Short title: CBT and VLQ

\title{
Valued living before and after CBT
}

Jürgen Hoyer1, Jasmin Čolić1, Gerd Grübler2, Andrew T. Gloster3

1Institute of Clinical Psychology and Psychotherapy, Technische Universitaet Dresden (Germany)

2Institute of Philosophy, Technische Universitaet Dresden (Germany)

3Clinical Psychology and Intervention Science, University of Basel (Switzerland)

Manuscript accepted in Journal of Contemporary Psychotherapy

Corresponding author:

Prof. Dr. Jürgen Hoyer

Technische Universität Dresden

Klinische Psychologie und Psychotherapie

Hohe Str. 53

D-01187 Dresden

juergen.hoyer@tu-dresden.de

phone: ++49-351-46336986, fax: ++49-351-46336955 


\begin{abstract}
(227 words)
Background. Whether and to what degree psychotherapy leads to changes in patients' valuebased actions is not well documented. In this study we examined whether cognitive behavioral therapy, without explicit values work, enhanced value-oriented action. We also explored the role of change in valued action for subsequent life satisfaction and continued change after therapy. Additionally, data on the reliability and validity of the Valued Living Questionnaire (VLQ) are reported.
\end{abstract}

Methods. We analyzed the pre-, post-, and 6-month-follow-up-data of 3,687 patients of a university psychotherapy outpatient clinic, most of which suffered from reliably diagnosed anxiety and mood disorders. Questionnaires included the VLQ (with 10 items each on the "importance" and "consistency" of values), symptom scales (Beck Depression Inventory; Brief Symptom Inventory), and the Satisfaction with Life Scale.

Results. Over the course of therapy significant improvements in value-oriented action were found $(d=.34)$, especially in treatment responders $(d=.51)$. Increase of value-oriented action significantly explained satisfaction with life at end of treatment, even after controlling for symptom reduction. Temporally preceding improvement on the VLQ predicted further symptom reduction until follow-up.

Conclusion. Data indicate that psychotherapy positively affects valued living, even when it is not explicitly targeted in treatment. Valued living may have a role in the course and maintenance of therapeutic change.

Keywords: Valued Living Questionnaire - valued action - psychometric properties treatment satisfaction - positive side effects 


\section{Introduction}

Personal values are abstract desirable goals that serve as guiding principles in peoples' lives (Kluckhohn, 1951). As such, they transcend specific circumstances and are important over time (Roccas \& Sagiv, 2010). Similarly, values have also been defined as "personal choices about what is important in life, which can guide the selection of behaviors and lead to satisfaction even in the absence of external reinforcement" (Berghoff, Forsyth, Ritzert, Eifert \& Anderson, p.1388). This definition makes clear that taking actions that are closely connected to personal values might be of central importance for psychological functioning (see, e.g., Vowles,McCracken, \&O'Brien, 2011) and well-being (Welzel \& Inglehart, 2010), a finding that makes the concept of values relevant also for clinical psychology and psychotherapy.

The role of values and of the degree to which people personally feel capable of living up to them has recently been particularly emphasized by proponents of Acceptance and Commitment Therapy (ACT; Hayes, Strosahl \& Wilson, 2012). . Several therapeutic interventions aiming at the clarification of values and at a better commitment to one's values have been proposed in ACT. Furthermore, ACT theory formulates assumptions about the role that the "activation of values" plays for human change in general. Most importantly, it has been postulated that changes in valued living can precede further changes, e.g., in symptom reduction (Gloster, Klotsche, et al., 2017). Apart from some recent exceptions (S. A. Hayes, Orsillo, \& Roemer, 2010; Wersebe et al., 2017), empirical data on change in valued living are rare, despite their potential to contribute to a better understanding of general therapeutic change processes.

One open question is to what extent increased valued living is a typical characteristic of any successful psychotherapy and not only of successful ACT as shown by Hayes et al. 
(2010) or Wersebe et al. (2017). A promotion of valued living is, for example, not explicitly intended within Cognitive Behavioral Therapy (CBT), which primarily aims at the reduction of clients' suffering by reducing the symptomatic behavior (see e.g. Craske, 2010; Gloster et al., 2017). Nevertheless, it can be argued that psychotherapeutic treatment (like CBT) impacts more than isolated symptoms as conceptualized by the classical medical model or other mechanistic explanations of human behavior (Hoyer, 2016). Psychotherapy can rather be assumed to affect patients' personal vulnerabilities and risk-factors (such as low selfesteem or low self-efficacy) in a generalized way and the corresponding changes might have broad impact on a person's psychological life situation. . In the case of CBT it can be assumed that it helps to reduce behavioral and experiential avoidance, teaches skills and competencies to solve problems and broadens the overall capacity to behave adaptively under stress (see, e.g., Craske, 2010). All these improvements can be expected to reduce the psychological barriers for the individual to more fully engage in their personally relevant values. Following this logic, we expected an increase in valued living to occur during or after successful psychotherapeutic treatment with CBT, even if no explicit strategies to clarify values or to enhance valued action (as in ACT) had been applied. Such an unspecific effect of psychotherapy (here: CBT) on valued living has, to our knowledge, not been previously tested.

Our second research question asks how important the increase in valued living is for the life satisfaction of psychotherapy patients.

Reflecting on claims that both positive mental health and psychopathology should be monitored in psychotherapy research (Cougle, 2012; Hoyer, 2016; Keyes, 2005; Trompetter, Lamers, , Westerhof, Fledderus \& Bohlmeijer, 2017)., we were interested in analyzing whether progress in symptom reduction and increase in valued living independently leads to enhanced satisfaction with life after psychotherapy. Since valued living as a concept is clearly 
distinguishable from symptoms and symptom reduction, it should independently contribute to the explanation of satisfaction with life after therapy if it is indeed important. More precisely, our second hypothesis postulates that increased valued living explains satisfaction with life after therapy even when the effects of symptom reduction are statistically controlled.

Our third research question was aimed at investigating whether improved valued living precedes symptom reduction. Typically, as posited in the so called consequence model, clinicians expect that changes in suffering precede changes in the other variables, e.g., in values, a process that was called remoralization, e.g., by Howard, Lueger, Maling, \& Martinovich, (1993). This position is contrasted by the antecedence model (equivalent to ACT theory; cf. S. C. Hayes et al., 1999) which posits that changes in experiential acceptance and valued action can temporally precede changes in suffering. Given that we could measure change in our study between treatment start (baseline, BL), treatment end (post-treatment, Post), and at 6-month follow-up (FU), a test of the antecedence model would implicate that changes of valued action between BL and Post precede change in suffering (as measured via symptom variables) that occurs only between Post and FU. This was our third hypothesis.

Summarizing, three assumptions about valued action and its function in psychotherapy, namely CBT, were tested: We assumed that valued living increases during CBT (Hypothesis 1), that these changes predict the satisfaction with life after therapy over and above the effects due to reduction of symptoms (Hypothesis 2), and that these changes predict subsequent changes in suffering that occur between end of treatment and follow-up (Hypothesis 3).

The strength of our tests, its internal validity, largely depends on the reliability and validity of the measures used. While we could use well-validated self-report measures for general psychopathology, depression, and satisfaction with life (see below), the reliability and 
validity of the measure for valued living, the Valued Living Questionnaire (VLQ; Wilson et al., 2010), has been studied less frequently in research contexts outside of ACT studies. Therefore, we will also report on the internal consistency and selected aspects of construct validity of VLQ.

\section{Methods}

\section{Study design and sample}

We analyzed the BL, Post, as well as FU data of $N=3,687$ patients of a university psychotherapy outpatient clinic collected between February 2004 and February 2015. All patients gave their written consent and were reliably diagnosed using the DSM-IV MunichComposite International Diagnostic Interview (M-CIDI; Wittchen \& Pfister, 1997). The time between BL and Post differed depending on the course of therapy. The average duration of treatment was $M=26.23$ sessions $(S D=18.27)$ and $M=267$ days $(S D=274.43)$. The FU took place six months after treatment completion. As we collected data from an outpatient clinic, and not within a controlled study, only $19.7 \%$ of the original sample $(n=727)$ completed the FU assessment. If FU was not returned by the patients as requested, no further measures to contact the patient were taken. The sociodemographic and clinical characteristics of the sample are shown in Table 1.

\section{Measures}

At BL, Post and FU participants were asked to complete the VLQ, the Beck Depression Inventory II (BDI-II, Beck, Steer, \& Brown, 1996; German Version: Hautzinger, Keller, \& Kühner, 2006), the Brief Symptom Inventory (BSI; Derogatis, 1993; German Version: Geisheim et al., 2002), and the Satisfaction with Life Scale (SWLS; Diener, Emmons, Larsen, \& Griffin, 1985; German Version: Glaesmer, Grande, Braehler, \& Roth, 2011). In the present study, the German versions of the instruments were used. 
VLQ. The VLQ focuses on ten central domains of human activity, namely: family, marriage/couple/intimate relationships, parenting, friendship, work, education, recreation, spirituality, community life, and physical wellbeing. On a scale from 1-10 (in the German version, on scale from 1-5) patients estimate how important these domains are for them and how consistently they pursue goals within these domains. This makes it possible to assess inconsistencies between importance and effort. From these scales, we calculated an average score for Importance (V-I) and Valued Action (V-A, i.e. consistency scale) across the domains. Also, a Valued Living Composite (VLQ-C) for each domain was calculated by multiplying its respective importance and consistency score. Preliminary studies (Wilson et al., 2010) showed good internal consistency for the V-I scale $(\alpha=.79-.83)$ and adequate internal consistency for the V-A scale $(\alpha=.58-.60)$ and the VLQ-C $(\alpha=.65-.74)$. However, since psychometric properties of the German version of the VLQ are lacking, we calculated them in the present study.

BDI-II. The BDI-II consists of 21 items. The items are rated on a four-point scale (03) and depict cognitive (e.g. self-dislike, pessimism, guilt) and somatic-affective dimensions (e.g. loss of energy, changes in appetite, fatigue) of depression (Steer, Ball, Ranieri, \& Beck, 1999). The total score can range from 0-63. The BDI-II showed high internal consistency ( $\alpha$ $=.92-.93)$ and high test-retest reliability ( $r=.93$; Beck et al., 1996).

BSI. The BSI is a short version of the symptom checklist SCL-90-R (Derogatis, 1977). It consists of 53 items that cover clinically relevant symptoms across nine dimensions: somatization, obsessive-compulsive, interpersonal sensitivity, depression, anxiety, hostility, phobic anxiety, paranoid ideation, and psychoticism. Four of the 53 items do not load on any dimension, but are included because of their clinical relevance (Derogatis, 1993). The items are rated on a five-point scale, reflecting the severity of distress from 0 (not at all) to 4 (extremely). The item scores can be summarized into three global indices. For the present 
study, we used the Global Severity Index (GSI), which represents the mean of the completed item scores. The GSI is only calculated if there are not more than 13 missing values overall (and >1 per dimension; Franke, 2000). For the German version, Cronbach's $\alpha$-coefficients of the dimensions ranged from .70 to .89, while the GSI had an $\alpha$ of .96 (Geisheim et al., 2002).

SWLS. The SWLS consists of five statements related to satisfaction with one's life (Diener et al., 1985). The Items are assessed on a 7-point scale that indicate the degree of agreement with the statements, ranging from 1 (strongly disagree) to 7 (strongly agree). The items are summed into a total score that can range from 5-35. The SWLS showed high internal consistency $(\alpha=.87)$ and good test-retest reliability $(r=.82)$

\section{Statistical analyses}

The statistical analyses were conducted using Stata Statistical Software Version 14.2. (StataCorp, 2015). Changes between BL, Post and FU were calculated using linear regression analyses with time being dummy-coded and modeled as discrete $(0=\mathrm{BL}, 1=$ Post, $2=\mathrm{FU})$. The effect sizes (ES) were derived as differences in means divided by the standard deviation at BL. In accordance with Cohen (1988), ES of 0.2 were interpreted as small, ES of 0.5 as medium and ES of 0.8 and bigger as large. This analysis was carried out for treatment responders as a sub-sample as well. Responders were patients that had a decrease of $47 \%$ in their BDI-II scores from BL to Post (Riedel et al., 2010). For Hypothesis 2 (H2) and 3 (H3), separate hierarchical regression analyses were conducted. For H2, SWLS at Post was used as outcome, while the BL scores of SWLS, GSI, BDI-II and VLQ-C were entered in the first step. In the second step the changes from BL to Post in BSI ( $\Delta$ GSIBL-Post) and in BDI-II $(\triangle \mathrm{BDI}$ BL-Post) were added as predictors, while in the third and final step the change in the VLQ Composite from BL to Post was entered ( $\triangle$ VLQ-CBL-Post). For H3, the changes from Post to FU in GSI ( $\Delta$ GSIPost-FU) and in BDI-II ( $\Delta$ BDIPost-FU) were used as outcomes, while the 
BL scores of BDI, GSI and VLQ-C were entered in the first step, and the $\triangle$ VLQ-CBL-Post in the second and final step. All predictors in the hierarchical regression analyses were centered at their means. In all analyses, the $p$-value was set to .05 .

\section{Results}

\section{Descriptive statistics}

Internal consistency of the VLQ. The scales had a comparatively low internal consistency, with $\alpha=.62$ for V-I, $\alpha=.67$ for V-A, and $\alpha=.66$ for VLQ-C. This was to be expected as the scales depicted in the VLQ cannot fully be regarded as homogenous traits but needs to be considered when interpreting the correlational findings with the VLQ reported below.

VLQ. At baseline most values were indicated as highly important ( $>4$ points on the 15 scale; see Figure 1). Only education/training, citizenship/community life and spirituality were considered less important $(<4)$. The values with the highest V-A scores were friends/social life and family (both >3), while other values had a V-A scored below three. The largest discrepancies were found for physical self-care and marriage/couples/intimate relationships (1.72 and 1.60), followed by work and recreation/fun (both 1.30).

We also analyzed in how many instances per patient there was a discrepancy between the importance of a value and its respective consistency at baseline. Only $n=13(0.35 \%)$ patients had no discrepancies in any of the domains, while $n=28(0.76 \%)$ had a discrepancy in only one life domain. A total of $n=73(1.98 \%)$ patients had two discrepancies, $n=119$ (3.23\%) had three discrepancies, $n=213(5.78 \%)$ had four discrepancies, and $n=372$ $(10.09 \%)$ had five discrepancies. A total of $77.81 \%$ of all patients $(n=2869)$ revealed discrepancies in more than five domains. The most frequent discrepancies were reported in 
the domains of physical self-care, fun/recreation and work, while the least discrepancies occurred in the domains of spirituality and citizenship (see also Fig. 1).

Means and standard deviations. The statistics of all measures at BL, Post and FU for the complete sample and for the subsample of responders are shown in Table 2 . The differences and effect sizes from BL to Post and to FU are depicted in Table 3.

Correlation analyses. There were medium to large correlations between the outcomes at BL. All correlations were significant (see Table 4).

\section{Hypothesis 1}

The scores of the VLQ showed significant increases from BL to Post and from BL to FU in the VLQ-C and V-A scores, as well as decreases in the V-I values. There were no differences from Post-FU. While the ES for V-I were small, the ES for V-A and VLQ-C were mostly moderate sized (Table 3). For the sub-sample of those showing clinically significant response with respect to their depressive symptoms, the ES were even stronger reaching medium size for V-A and VLQ-C (Table 3). In sum, Hypothesis 1 could be confirmed: CBT increases valued living.

\section{Hypothesis 2}

As the hierarchical regression analysis shows (see Table 5), increases in VLQ-C predict life satisfaction over and above the effects of mere symptom reduction. They explained an additional $3.74 \%$ of the variability in SWLS at end of treatment (as indicated by the $\Delta R_{2}$ in Block 3). The final model overall explained $62.9 \%$ of the variance. These results confirm Hypothesis 2.

\section{Hypothesis 3}


As the results show, the changes in VLQ that have occurred during therapy did predict subsequent change from Post to FU in GSI, as well as BDI (see Table 6). This confirms our third hypothesis: Increases in valued living precede the reduction of symptoms.

\section{Discussion}

In this naturalistic study we observed that patients report an increase in valued living during CBT (confirming Hypothesis 1), that these changes predicted the satisfaction with life after therapy over and above the effects due to reduction of symptoms (confirming Hypothesis 2), and that these changes predicted subsequent changes in suffering that occur between end of treatment and follow-up (confirming Hypothesis 3). These findings contradict models of change that assume symptom reduction to be a prerequisite for improved life satisfaction of patients. They are rather consistent with the idea that also change in valued action has a role for subsequent life satisfaction and continued change after therapy.

Furthermore, descriptive statistical analyses of the VLQ indicated that the items reflect domains that matter to people in that seven out of the 10 items included in the VLQ were rated as important (rated on average at least 4 on a five-point scale). At the same time, the VLQ demonstrated its potential to help clarify which values are consistently met or not as more than $99 \%$ of the respondents indicated to not live up fully to one or more values. This finding also signals that perfectly living up to the own values is an ideal which can usually not be met in life.

Results further show that CBT helps to increase valued living - even assuming that no specific techniques were applied to provoke this effect. However, given that we could not conduct empirical adherence analyses of the therapeutic interventions used during session based expert ratings, we cannot rule out that some therapists might have used some kind of values work in their session as the concept of values has become more prominent in the past 
years. Our findings can therefore only be interpreted as preliminary evidence for the assumption that indeed different types of psychotherapy have the potential to influence the amount of valued behavior a person realizes in life, no matter if this is an explicit aim of the therapy style (as is the case in ACT) or rather a "positive side effect" (Hoyer, 2016) of the therapeutic measures taken to systematically change approach and avoidance tendencies of patients. It is an interesting scientific question for the future to what extent ACT would outperform conventional CBT in direct comparison studies in terms of enhancement of valued living and which further consequences of these potential differences between the two therapeutic strategies will be observable in longitudinal investigations.

ACT itself does not claim to outperform other therapeutic approaches in terms of symptom reduction and does not do so in conscientiously conducted RCTs (e.g. Arch et al., 2012). ACT rather focuses on the reduction of struggling with given life circumstances. Following ACT logic, we argued that an increase in valued living would lead not only to symptom reduction (e.g., as valued action can include reduced avoidance), but also to heightened satisfaction with life. Although the symptom reduction did explain a relevant portion of the variance of satisfaction with life after therapy, increase of valued living independently explained additional variance. However, it has to be taken into account here that the treatment was not delivered in a short-term intense form but rather applied over longer time periods. This makes it possible that other more imminent life events might shape self-ratings of life satisfaction and that, in other words, therapeutic effects may only be among many others to explain satisfaction with life.

According to the antecedence model underlying ACT, we hypothesized that gains in valued living precede subsequent symptom reduction. Would those who improved their valued living during therapy show more subsequent reduction of general psychopathological symptoms and depression in the follow-up period? There was indication for such an effect in 
these data. This suggests that valued action has not only a buffer effect against further symptoms (and/or its relapse), but the engagement in valued living at one time has an advantageous effect on symptoms in the future. This is theoretically relevant because it runs counter to the implicit assumptions, teachings, and recommendations inherent in Western psychology, psychiatry, medicine, and even the self-help industry. Whereas reduction or elimination of uncomfortable symptoms is certainly desirable, their reduction or elimination is not a necessary condition for living life in a way that patients choose.

A number of limitations need to be clarified. Starting with the psychometric properties of our measure of valued living, the VLQ has conceptual and psychometric limitations as previous articles have criticized (Gloster, Klotsche, et al., 2017; Smout, Davies, Burns, \& Christie, 2014). While the measure has good face validity and clinical relevance, the internal consistency was unsatisfying in terms of classical test theory and the relatively low reliability coefficients might have led to underestimations of the correlation coefficients reported. Furthermore, our naturalistic study used data from just three different time points, giving us only a very rough basis for analyzing temporal patterns of change. In order to model the process of change more detailed it would be good to have studies acquiring data at much more time points. The temporal relations between valued living and symptom measures during the course of therapy should rather be analyzed on a session-to-session or, even better, on a moment-to-moment basis during the session. Another limitation is the number of initial patients that responded to our inquiries at follow-up, which could implicate a possible selection bias with reference to Hypothesis 3 . This concern is mitigated somewhat in that comparisons between the complete sample and those that responded to follow up did not reveal different patterns at other time points. Finally, all our data were gained by self-report instruments. Thus, we do not have objective, external information whether there was a real, observable change in valued action. Further research might include more real-life information 
in order to estimate the changes in the patients' life more precisely (Gloster, Miché, et al., 2017).

Despite these limitations our data demonstrate that inclusion of valued living is one good example for broadening the scope of what constitutes a good outcome in psychotherapy (see Rønnestad et al., 2018). Furthermore, we see our study as an important support for the assumption that valued living changes in other therapies than ACT as well, and has further effects on other important life domains - an idea that is worth investigating in further studies. 


\section{References}

Arch, J. J., Eifert, G. H., Davies, C., Vilardaga, J. C. P., Rose, R. D., \& Craske, M. G. (2012). Randomized clinical trial of cognitive behavioral therapy (CBT) versus acceptance and commitment therapy (ACT) for mixed anxiety disorders. Journal of Consulting and Clinical Psychology, 80(5), 750-765. http://dx.doi.org/10.1037/a0028310

Beck, A. T., Steer, R. A., \& Brown, G. K. (1996). Beck depression inventory-II (BDI-II). San Antonio, TX: The Psychological Corporation.

Berghoff, C. R., Forsyth, J. P., Ritzert, T. R., Eifert, G. H., \& Anderson, D. A. (2018). Evaluation of the contribution of values clarification to a brief mindfulness meditation intervention for anxiety. Journal of Clinical Psychology, 74(9), 1387-1402. doi:doi:10.1002/jclp.22610

Cohen, J. (1988). Statistical Power Analysis for the Behavioral Sciences (2nd ed.). Hillsdale, NJ: Lawrence Erlbaum Associates.

Cougle, J. R. (2012). What makes a quality therapy? A consideration of parsimony, ease, and efficiency. Behavior Therapy, 43(3), 468-481. https://doi.org/10.1016/j.beth.2010.12.007

Craske, M. G. (2010). Cognitive-behavioral therapy. Washington, DC: American Psychological Association.

Derogatis, L. R. (1977). The SCL-90 Manual I: Scoring, administration and procedures for the SCL-90. Baltimore, MD: Clinical Psychometric Research.

Derogatis, L. R. (1993). BSI Brief Symptom Inventory. Administration, Scoring, and Procedures Manual (4th Ed.). Minneapolis, MN: National Computer Systems. 
Diener, E., Emmons, R. A., Larsen, R. J., \& Griffin, S. (1985). The Satisfaction With Life Scale. Journal of Personality Assessment, 49(1), 71-75. https://doi.org/10.1207/s15327752jpa4901_13

Erekson, D. M., Lambert, M. J., \& Eggett, D. L. (2015). The relationship between session frequency and psychotherapy outcome in a naturalistic setting. Journal of Consulting and Clinical Psychology, 83(6), 1097-1107. http://dx.doi.org/10.1037/a0039774

Franke, G. H. (2000). Brief Symptom Inventory (BSI) von L.R. Derogatis (Kurzform der SCL90-R). Göttingen, Germany: Beltz.

Geisheim, C., Hahlweg, K., Fiegenbaum, W., Frank, M., Schröder, B., \& von Witzleben, I. (2002). Das Brief Symptom Inventory (BSI) als Instrument zur Qualitätssicherung in der Psychotherapie. Diagnostica, 48(1), 28-36. https://doi.org/10.1026//00121924.48.1.28

Glaesmer, H., Grande, G., Braehler, E., \& Roth, M. (2011). The German version of the Satisfaction With Life Scale (SWLS). European Journal of Psychological Assessment, 27(2), 127-132. https://doi.org/10.1027/1015-5759/a000058

Gloster, A. T., Klotsche, J., Ciarrochi, J., Eifert, G., Sonntag, R., Wittchen, H.-U., \& Hoyer, J. (2017). Increasing valued behaviors precedes reduction in suffering: Findings from a randomized controlled trial using ACT. Behaviour Research and Therapy, 91, 6471. https://doi.org/10.1016/j.brat.2017.01.013

Gloster, A. T., Miché, M., Wersebe, H., Mikoteit, T., Hoyer, J., Imboden, C., ... Lieb, R. (2017). Daily fluctuation of emotions and memories thereof: design and methods of an experience sampling study of major depression, social phobia, and controls. International Journal of Methods in Psychiatric Research, 26(3), n/a-n/a. https://doi.org/10.1002/mpr.1578 
Hautzinger, M., Keller, F., \& Kühner, C. (2006). Beck Depressions-Inventar (BDI-II). Frankfurt am Main, Germany: Harcourt Test Services.

Hayes, S. A., Orsillo, S. M., \& Roemer, L. (2010). Changes in proposed mechanisms of action during an acceptance-based behavior therapy for generalized anxiety disorder. Behaviour Research and Therapy, 48(3), 238-245. https://doi.org/10.1016/j.brat.2009.11.006

Hayes, S. C., Strosahl, K. D., \& Wilson, K. G. (1999). Acceptance and commitment therapy: An experiential approach to behavior change. New York, NY: Guilford Press.

Howard, K. I., Lueger, R. J., Maling, M. S., \& Martinovich, Z. (1993). A phase model of psychotherapy outcome: Causal mediation of change. Journal of Consulting and Clinical Psychology, 61(4), 678-685. http://dx.doi.org/10.1037/0022-006X.61.4.678

Hoyer, J. (2016). „Positive Nebenwirkungen“ von Psychotherapie. Zeitschrift für Klinische Psychologie und Psychotherapie, 45(3), 163-173. https://doi.org/10.1026/1616$3443 / \mathrm{a} 000370$

Keyes, C. L. M. (2005). Mental illness and/or mental health? Investigating axioms of the complete state model of health. Journal of Consulting and Clinical Psychology, 73, 539-548. DOI: 10.1037/0022-006X.73.3.539

Kluckhohn, C. (1951). Values and value-orientations in the theory of action: An exploration in definition and classification. In T. Parsons \& E. Shils (Eds.), Toward a General Theory of Action (pp. 388-433). Cambridge, MA: Harvard University Press.

Riedel, M., Möller, H.-J., Obermeier, M., Schennach-Wolff, R., Bauer, M., Adli, M., ... Seemüller, F. (2010). Response and remission criteria in major depression - A validation of current practice. Journal of Psychiatric Research, 44(15), 1063-1068. https://doi.org/10.1016/j.jpsychires.2010.03.006 
Roccas, S., \& Sagiv, L. (2010). Personal values and behavior: Taking the cultural context into account. Social and Personality Psychology Compass, 4, 30-41. doi:doi:10.1111/j.1751-9004.2009.00234.x

Rønnestad, M. H., Nissen-Lie, H. A., Oddli, H. W., Benum, K., Ekroll, V. B., Gullestad, S. E., ... Halvorsen, M. S. (2018). Expanding the Conceptualization of Outcome and Clinical Effectiveness. Journal of Contemporary Psychotherapy. https://doi.org/10.1007/s10879-018-9405-z

Smout, M., Davies, M., Burns, N., \& Christie, A. (2014). Development of the Valuing Questionnaire (VQ). Journal of Contextual Behavioral Science, 3(3), 164-172. https://doi.org/10.1016/j.jcbs.2014.06.001

StataCorp. (2015). Stata Statistical Software: Release 14. College Station, TX: StataCorp LP.

Steer, R. A., Ball, R., Ranieri, W. F., \& Beck, A. T. (1999). Dimensions of the Beck depression inventory-II in clinically depressed outpatients. Journal of Clinical Psychology, 55(1), 117-128. https://doi.org/10.1002/(SICI)10974679(199901)55:1<117::AID-JCLP12>3.0.CO;2-A

Trompetter, H. R., Lamers, S. M. A., Westerhof, G. J., Fledderus, M., \& Bohlmeijer, E. T. (2017). Both positive mental health and psychopathology should be monitored in psychotherapy: Confirmation for the dual-factor model in acceptance and commitment therapy. Behaviour Research and Therapy, 91, 58-63. doi:http://dx.doi.org/10.1016/j.brat.2017.01.008

Vowles, K. E., McCracken, L. M., \& O'Brien, J. Z. (2011). Acceptance and values-based action in chronic pain: A three-year follow-up analysis of treatment effectiveness and process. Behaviour Research and Therapy, 49, 748-755. https://doi.org/10.1016/j.brat. 2011.08.002 
Welzel, C., \& Inglehart, R. (2010). Agency, values, and well-being: A human development model. Social Indicators Research, 97, 43-63. DOI 10.1007/s11205-009-9557-z

Wersebe, H., Lieb, R., Meyer, A. H., Hoyer, J., Wittchen, H.-U., \& Gloster, A. T. (2017). Changes of valued behaviors and functioning during an Acceptance and Commitment Therapy Intervention. Journal of Contextual Behavioral Science, 6(1), 63-70. https://doi.org/10.1016/j.jcbs.2016.11.005

Wilson, K. G., Sandoz, E. K., Kitchens, J., \& Roberts, M. (2010). The Valued Living Questionnaire: Defining and Measuring Valued Action Within a Behavioral Framework. Psychological Record, 60(2), 249-272. https://doi.org/10.1007/BF03395706

Wittchen, H.-U., \& Pfister, H. (1997). DIA-X-Interviews: Manual für Screening-Verfahren und Interview; Interviewheft Längsschnittuntersuchung (DIA-X-Lifetime); Ergänzungsheft (DIA-X-Lifetime); Interviewheft Querschnittuntersuchung (DIA-X-12 Monate); Ergänzungsheft (DIA-X-12Monate); PC-Programm zur Durchführung des Interviews (Längs- und Querschnittuntersuchung); Auswertungsprogramm. Frankfurt: Swets \& Zeitlinger 


\section{Tables}

Table 1. Sociodemographic and clinical characteristics of the sample [Link back to sample]

\begin{tabular}{lr} 
Characteristics & Descriptives \\
\hline Age, $M(S D)$ & $35.28(13.27)$ \\
Female, $\%$ & 65.69
\end{tabular}

Years of education, $\%$

$8-10$

$11-13$

$14+$

In relationship, yes \%

Primary diagnoses, $\%$

F0

F1

F2

0.77

F3

34.74

F4

46.88

F5

8.84

F6

Other

0.49

Number of diagnoses, $\%$

2

Note . F0 = Organic mental disorders; F1 = Disorders due to psychoactive substance use; F2 = Schizophrenia, schizotypal and delusional disorders; F3 = Affective disorders; F4 = Anxiety, stress-related and somatoform disorders; F5 = Behavioral syndromes associated with physiological disturbances and physical factors; F6 = Disorders of adult personality 
Table 2. VLQ, GSI, BDI and SWLS scores at Baseline, Post and FU in the complete sample $(N=3687)$ and responders only $(n=849)[$ Back to Results]

\begin{tabular}{lcccccc}
\hline Variables & \multicolumn{3}{c}{ Complete sample } & \multicolumn{3}{c}{ Responders only } \\
& BL & Post & FU & BL & Post & FU \\
& $M(S D)$ & $M(S D)$ & $M(S D)$ & $M(S D)$ & $M(S D)$ & $M(S D)$ \\
\hline VLQ & & & & & & \\
V-I & $3.95(0.49)$ & $3.92(0.49)$ & $3.90(0.49)$ & $4.02(0.44)$ & $3.95(0.45)$ & $3.94(0.47)$ \\
V-A & $2.86(0.62)$ & $3.12(0.62)$ & $3.19(0.64)$ & $2.89(0.57)$ & $3.26(0.56)$ & $3.31(0.57)$ \\
VLQ-C & $11.87(3.14)$ & $12.94(3.25)$ & $13.11(3.31)$ & $12.09(2.88)$ & $13.56(2.99)$ & $13.65(3.02)$ \\
GSI & $1.08(0.65)$ & $0.52(0.54)$ & $0.51(0.52)$ & $1.05(0.60)$ & $0.32(0.34)$ & $0.37(0.39)$ \\
BDI-II & $17.16(10.40)$ & $8.35(8.56)$ & $8.91(9.17)$ & $17.52(9.44)$ & $4.47(5.07)$ & $6.33(6.92)$ \\
SWLS & $17.23(6.99)$ & $21.56(7.00)$ & $21.58(7.25)$ & $17.63(6.88)$ & $23.60(5.98)$ & $23.09(6.63)$ \\
\hline
\end{tabular}

Note. VLQ = Valued Living Questionnaire; V-I = Values Importance (Range 1-5); V-A = Valued Action (Range 1-5); VLQ-C = Valued Living Questionnaire Composite (Range 1-25); GSI = Global Severity Index (Range 0-4); BDI-II = Beck-Depression Inventory - II (Range 0-63); SWLS $=$ Satisfaction with Life Scale (Range 0-25); BL = Baseline, Pre-treatment; Post = Post-treatment; FU = Follow-up 
Table 3. Within-group differences and effect sizes in VLQ, GSI, BDI and SWLS for the complete sample and for responders [Back to Results].

\begin{tabular}{|c|c|c|c|c|c|c|c|}
\hline \multirow{2}{*}{ Variables } & \multirow{2}{*}{ Differences } & \multicolumn{3}{|c|}{ Complete sample } & \multicolumn{3}{|c|}{ Responders only } \\
\hline & & $t$ & $p$ & $\mathrm{ES}[95 \% \mathrm{CI}]$ & $t$ & $p$ & $\mathrm{ES}[95 \% \mathrm{CI}]$ \\
\hline \multirow[t]{3}{*}{ V-I } & BL-Post & -2.80 & $<.01$ & $-0.08[-0.14,-0.02]$ & -3.31 & $<.01$ & $-0.16[-0.26,-0.06]$ \\
\hline & BL-FU & -3.26 & $<.01$ & $-0.13[-0.21,-0.05]$ & -3.24 & $<.01$ & $-0.19[-0.31,-0.08]$ \\
\hline & Post-FU & 0.96 & $=.34$ & $0.04[-0.05,0.13]$ & 0.51 & $=.61$ & $0.03[-0.08,0.14]$ \\
\hline \multirow[t]{3}{*}{$\mathrm{V}-\mathrm{A}$} & BL-Post & 13.57 & $<.01$ & $0.42[0.37,0.49]$ & 13.40 & $<.01$ & $0.64[0.55,0.74]$ \\
\hline & BL-FU & 13.04 & $<.01$ & $0.53[0.45,0.61]$ & 12.60 & $<.01$ & $0.73[0.62,0.85]$ \\
\hline & Post-FU & -2.23 & $=.02$ & $-0.10[-0.19,-0.01]$ & -1.57 & $=.12$ & $-0.09[-0.21,0.02]$ \\
\hline \multirow[t]{3}{*}{ VLQ-C } & BL-Post & 10.67 & $<.01$ & $0.34[0.28,0.40]$ & 10.31 & $<.01$ & $0.51[0.41,0.61]$ \\
\hline & BL-FU & 9.59 & $<.01$ & $0.40[0.31,0.48]$ & 9.03 & $<.01$ & $0.54[0.42,0.66]$ \\
\hline & Post-FU & -1.17 & $=.24$ & $-0.05[-0.15,0.03]$ & -0.53 & $=.59$ & $-0.03[-0.15,0.08]$ \\
\hline \multirow[t]{3}{*}{ GSI } & BL-Post & -36.15 & $<.01$ & $-0.85[-0.90,-0.80]$ & -40.64 & $<.01$ & $-1.21[-1.27,-1.15]$ \\
\hline & BL-FU & -30.98 & $<.01$ & $-0.87[-0.93,-0.82]$ & -32.89 & $<.01$ & $-1.13[-1.19,-1.06]$ \\
\hline & Post-FU & 0.63 & $=.53$ & $0.02[-0.04,0.08]$ & -2.53 & $=.01$ & $-0.08[-0.15,-0.02]$ \\
\hline \multirow[t]{3}{*}{ BDI } & BL-Post & -70.82 & $<.01$ & $-0.77[-0.79,-0.75]$ & -46.15 & $<.01$ & $-1.38[-1.44,-1.32]$ \\
\hline & BL-FU & -76.98 & $<.01$ & $-0.84[-0.86,-0.82]$ & -34.32 & $<.01$ & $-1.18[-1.25,-1.11]$ \\
\hline & Post-FU & 6.15 & $<.01$ & $0.07[0.05,0.09]$ & -5.84 & $<.01$ & $-0.20[-0.26,-0.13]$ \\
\hline \multirow[t]{3}{*}{ SWLS } & BL-Post & 24.19 & $<.01$ & $0.61[0.57,0.67]$ & 23.79 & $<.01$ & $0.63[0.58,0.68]$ \\
\hline & BL-FU & 20.33 & $<.01$ & $0.62[0.56,0.68]$ & 18.86 & $<.01$ & $0.58[0.52,0.64]$ \\
\hline & Post-FU & -0.10 & $=.92$ & $-0.00[-0.07,0.06]$ & 1.81 & $=.07$ & $0.05[-0.01,0.11]$ \\
\hline
\end{tabular}

Note. V-I = Values Importance $($ Range 1-10); V-A = Valued Action (Range 1-10); VLQ-C = Valued Living Questionnaire Composite (Range 10-100); GSI = Global Severity Index (Range 0-4); BDI-II = Beck-Depression Inventory - II (Range 0-63); SWLS = Satisfaction with Life Scale (Range 0-25); BL = Baseline, Pre-treatment; Post $=$ Post-treatment; FU = Follow-up; ES = Effect size; CI $=$ Confidence Interval. Significant p-values are bold. 
Table 4. Correlations between outcomes at baseline [Back to Results].

\begin{tabular}{ccccc}
\hline & VLQ-C & GSI & BDI-II & SWLS \\
\hline VLQ-C & $\mathrm{r}(3685)=1$ & & & \\
$p$ & $<.001$ & & & \\
GSI & $\mathrm{r}(3665)=-.34$ & $\mathrm{r}(5177)=1$ & & \\
$p$ & $<.001$ & $<.001$ & & \\
BDI-II & $\mathrm{r}(3685)=-.41$ & $\mathrm{r}(5177)=.72$ & $\mathrm{r}(5353)=1$ & \\
$p$ & $<.001$ & $<.001$ & & $<.001$ \\
SWLS & $\mathrm{r}(3643)=.56$ & $\mathrm{r}(4925)=-.48$ & $\mathrm{r}(5097)=-.57$ & $\mathrm{r}(5100)=1$ \\
$p$ & $<.001$ & $<.001$ & $<.001$ & \\
\hline
\end{tabular}

Note. VLQ-C = Valued Living Composite; GSI = Global Severity Index; BDI-II = Beck

Depression Inventory II; SWLS = Satisfaction with life scale 
Table 5. Summary of hierarchical regression analysis for variables predicting satisfaction with life at end of treatment [Back to Results].

\begin{tabular}{|c|c|c|c|c|c|c|c|c|c|}
\hline \multirow[t]{2}{*}{ Predictors } & \multicolumn{3}{|l|}{ Block 1} & \multicolumn{3}{|l|}{ Block 2} & \multicolumn{3}{|l|}{ Block 3} \\
\hline & $B$ & $S E_{B}$ & $\beta$ & $B$ & $S E_{B}$ & $\beta$ & $B$ & $S E_{B}$ & $\beta$ \\
\hline SWLS-BL & 0.46 & 0.03 & $0.45 * *$ & 0.46 & 0.02 & $0.45 * *$ & 0.43 & 0.02 & $0.42 * *$ \\
\hline BDI-II-BL & -0.08 & 0.03 & $-0.11 * *$ & -0.27 & 0.03 & $-0.36 * *$ & -0.22 & 0.03 & $-0.29 * *$ \\
\hline GSI-BL & 0.48 & 0.40 & 0.04 & -1.64 & 0.48 & $-0.14 * *$ & -1.58 & 0.46 & $-0.14 * *$ \\
\hline VLQ-C-BL & 0.27 & 0.67 & $0.11 * *$ & 0.16 & 0.05 & $0.07 * *$ & 0.42 & 0.05 & $0.18 * *$ \\
\hline$\Delta$ GSIBL-Post & & & & 3.48 & 0.49 & $0.30 * *$ & 2.90 & 0.47 & $0.25 * *$ \\
\hline$\Delta$ BDIBL-Post & & & & 0.30 & 0.03 & $0.40 * *$ & 0.25 & 0.03 & $0.33 * *$ \\
\hline$\Delta$ VLQ-CBL-Post & & & & & & & -0.59 & 0.05 & $-0.23 * *$ \\
\hline$\Delta R_{2}$ & & & - & & & .269 & & & .037 \\
\hline$R_{2}$ & & & .323 & & & .592 & & & .629 \\
\hline$F$ for $\Delta R 2$ & & $\mathrm{~F}(4,12$ & $150.54 * *$ & & $\mathrm{~F}(2,12$ & $414.82 * *$ & & $\mathrm{~F}(1,12$ & $126.88 * *$ \\
\hline
\end{tabular}

Note. SWLS-BL = Satisfaction with life scale at baseline; BDI-II-BL = Beck Depression Inventory II at baseline; GSI-BL = Global Severity Index at baseline; VLQ-C-BL = Valued Living Composite at baseline; $\triangle$ GSIBL-Post $=$ Change in Global Severity Index from baseline to posttreatment; $\triangle \mathrm{BDIBL}$-Post $=$ Change in Beck Depression Inventory II from baseline to post-treatment. All predictors were mean-centered.

Significant predictors in each block are bold.

$* p<.05, * * p<.01$ 
Table 6. Summary of hierarchical regression analysis for variables predicting the change in Global Severity Index and in the Beck Depression Inventory II from post-treatment to follow-up [Back to Results]

\begin{tabular}{|c|c|c|c|c|c|c|c|c|c|c|c|c|}
\hline \multirow{3}{*}{ Predictors } & \multicolumn{6}{|c|}{$\Delta$ GSIPost-FU } & \multicolumn{6}{|c|}{$\Delta$ BDIPost-FU } \\
\hline & \multicolumn{3}{|l|}{ Block 1} & \multicolumn{3}{|c|}{ Block 2} & \multicolumn{3}{|l|}{ Block 1} & \multicolumn{3}{|l|}{ Block 2} \\
\hline & $B$ & $S E_{B}$ & $\beta$ & $B$ & $S E_{B}$ & $\beta$ & $B$ & $S E_{B}$ & $\beta$ & $B$ & $S E_{B}$ & $\beta$ \\
\hline GSI-BL & -0.04 & 0.03 & -0.07 & -0.03 & 0.03 & -0.07 & -0.77 & 0.55 & -0.08 & -0.74 & 0.55 & -0.08 \\
\hline BDI-II-BL & 0.00 & 0.00 & 0.09 & 0.00 & 0.00 & 0.08 & 0.00 & 0.04 & 0.00 & -0.00 & 0.04 & -0.01 \\
\hline VLQ-C-BL & 0.01 & 0.01 & $0.09 *$ & 0.01 & 0.01 & 0.05 & 0.06 & 0.08 & 0.03 & -0.00 & 0.09 & -0.00 \\
\hline$\Delta$ VLQ-CBL-Post & & & & 0.01 & 0.01 & $0.10 *$ & & & & 0.17 & 0.08 & $0.09 *$ \\
\hline$\Delta R_{2}$ & & & - & & & .008 & & & - & & & .006 \\
\hline$R_{2}$ & & & .010 & & & .018 & & & .010 & & & .016 \\
\hline$F$ for $\Delta R 2$ & & $\mathrm{~F}(3,6$ & $=1.97$ & & $\mathrm{~F}(1,6$ & $=4.93^{*}$ & & $\mathrm{~F}(3,6$ & $=1.96$ & & $\mathrm{~F}(1,6$ & $=3.95^{*}$ \\
\hline
\end{tabular}

Note. $\Delta \mathrm{GSIPost-FU}=$ Change in Global Severity Index from post-treatment to follow-up; $\Delta \mathrm{BDIPost-FU}=$ Change in Beck Depression Inventory II from post-treatment to follow-up; GSI-BL = Global Severity Index at baseline; BDI-II-BL = Beck Depression Inventory II at baseline; VLQ$\mathrm{C}-\mathrm{BL}=$ Valued Living Composite at baseline; $\triangle \mathrm{VLQ}-\mathrm{CBL}_{\mathrm{BL}}$-Pst $=$ Change in Valued Living Composite from baseline to post-treatment. All predictors are mean-centered. Significant predictors in each block are bold.

$* p<.05, * * p<.01$ 


\section{Figures}

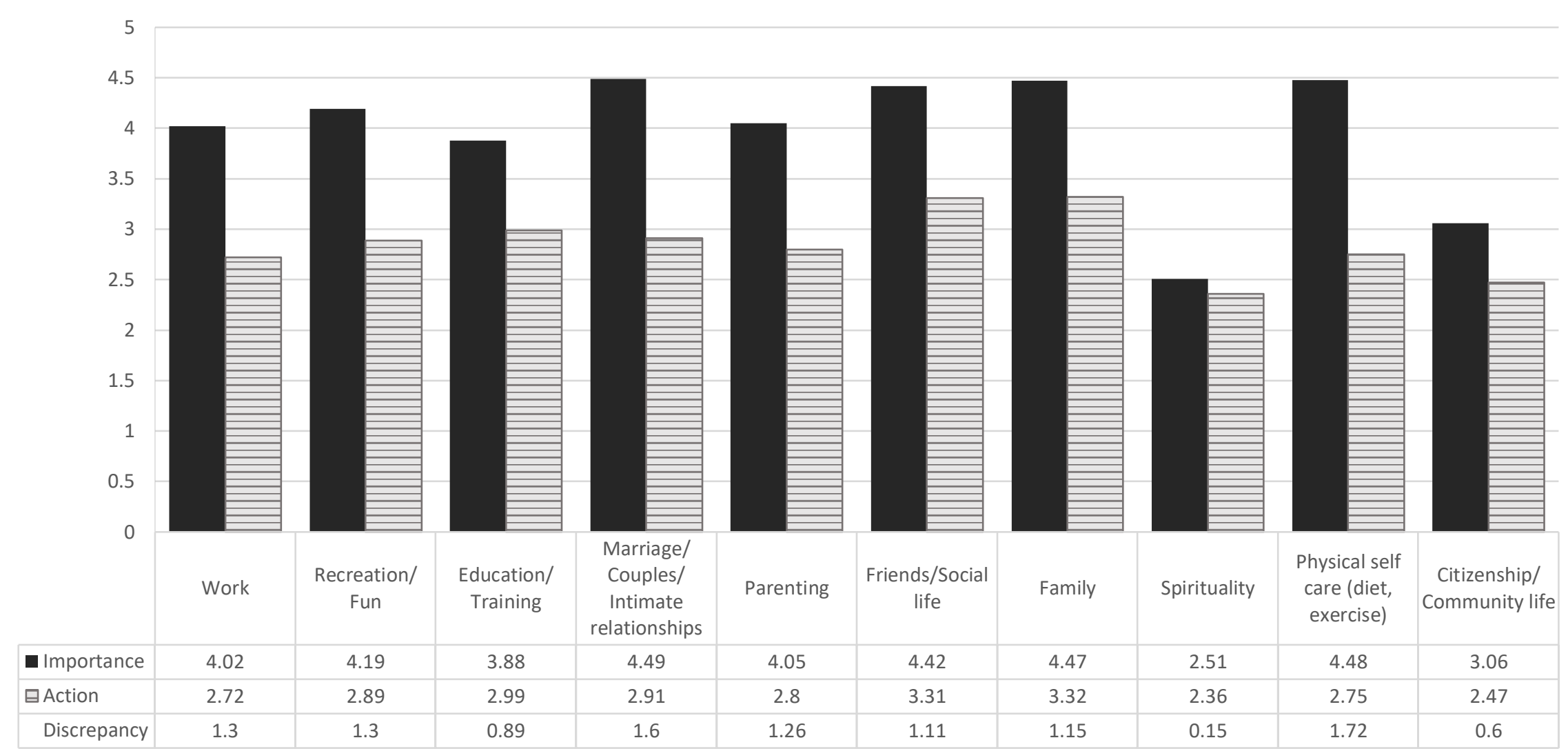

Figure 1. Mean importance, action/consistency and discrepancy of individual values. [Back to Results]. 